\title{
Genetic variation and constraints on the evolution of defense against spittlebug (Philaenus spumarius) herbivory in Mimulus guttatus
}

\author{
CT Ivey ${ }^{1}$, DE Carr ${ }^{2}$ and MD Eubanks ${ }^{3}$ \\ ${ }^{1}$ Department of Biological Sciences, California State University, Chico, CA, USA; ${ }^{2 B}$ landy Experimental Farm, University of Virginia, \\ Boyce, VA, USA and ${ }^{3}$ Department of Entomology, Texas A and M University, College Station, TX, USA
}

\begin{abstract}
Plants mediate carbon into most ecosystems and are thus under persistent attack by diverse enemies. The evolution of defense against such assaults will depend on the availability of genetic variation, as well as the costs and constraints on defense. We estimated the magnitude of genetic variation for defense against spittlebug (Philaenus spumarius) herbivory in Mimulus guttatus using a diallel cross-grown in a greenhouse. Except for flowering time, additive genetic variation for the plant traits we measured was negligible, regardless of herbivory environment. In contrast, nonadditive genetic variation contributed significantly to all plant traits measured. We found significant additive genetic variation among plants for biomass of adult spittlebugs, suggesting heritability for resistance to herbivory. The other putative resistance trait measured, spittlebug maturation time, was not significantly heritable. We found no evidence for significant genetic
\end{abstract}

variation for tolerance to herbivory except for a small nonnuclear paternal contribution to tolerance for flower number. Additive genetic correlations indicated that more resistant plant genotypes (in terms of adult spittlebug biomass) were also smaller in the absence of spittlebugs, suggesting a potential cost of resistance to herbivory. We found no other significant genetic correlations indicating a cost of defense, nor did we find evidence for a tradeoff between resistance and tolerance to herbivory. Overall, these results suggest the future adaptive evolution of tolerance to spittlebugs in this population will be limited primarily by available genetic variation, whereas the future evolution of antibiosis resistance may be constrained by allocation costs of resistance.

Heredity (2009) 102, 303-311; doi:10.1038/hdy.2008.122; published online 17 December 2008

Keywords: diallel; genetic correlation; herbivory; Mimulus guttatus; resistance; tolerance

\section{Introduction}

Plants are the primary source of carbon and energy for most other organisms, and as a consequence, they are under persistent attack by natural enemies. A wide variety of strategies for limiting the impact of consumers can be found among plants. These defense phenotypes have been broadly categorized into two forms. 'Resistance' includes traits that limit enemy attack (antixenosis) or performance (antibiosis), whereas 'tolerance' describes traits that affect the performance of plants under attack (Strauss and Agrawal, 1999).

In general, some form of defense is expected to evolve in response to attack by an enemy if there is a fitness cost exacted by the attack. In the absence of genetic variation for traits conferring resistance or tolerance, however, defense will not evolve. In addition, the evolution of defensive traits may be constrained if their development requires resources that would otherwise contribute toward growth or reproduction (Strauss et al., 2002). Under such circumstances some specialization in defense

Correspondence: Dr CT Ivey, Department of Biological Sciences, California State University, Chico, 205 Holt Hall, Chico, CA, 95929-0515, USA.

E-mail: ctivey@csuchico.edu

Received 19 June 2008; revised 22 October 2008; accepted 11 November 2008; published online 17 December 2008 strategy is expected (van der Meijden et al., 1988). Selection for tolerance, for example, is expected to be weak on a highly resistant genotype, because the two forms of defense are considered to be redundant (Fineblum and Rausher, 1995). The evolution of defense, therefore, can be constrained by low genetic variation or by genetic correlations with other traits on which selection acts in opposition.

A number of earlier studies have contributed toward our understanding of genetic variation and constraints in herbivore defense by using clonal (Marquis, 1990; Wise, 2007) or half-sib (Maddox and Root, 1987; Fornoni et al., 2003) experimental designs (reviewed in Berenbaum and Zangerl, 1992; Kennedy and Barbour, 1992). These approaches, however, cannot partition genetic variation from most nonadditive sources, such as epistatic or dominance variation, or non-nuclear parental effects (Lynch and Walsh, 1998). Any substantial contribution by these other sources of genetic variation could inflate the estimate of heritability in such studies and lead to misinterpretation of the potential response of traits to natural selection. Nonadditive genetic variation for defense is routinely reported for crop species (Soper et al., 1984; Wilson, 1990; Dhliwayo et al., 2005), but very few studies of defense in natural systems have used experimental designs that can reveal nonadditive genetic variation (but see Simms and Rausher, 1987; Juenger and Bergelson, 2000). 
We used a diallel breeding design to estimate genetic variation and constraints on the evolution of resistance (antibiosis) and tolerance to herbivory by spittlebugs (Philaenus spumarius L., Cercopidae:Homoptera) in a population of yellow monkey flower (Mimulus guttatus DC, Phrymaceae, formerly Scrophulariaceae). The primary aims of our study were (1) to estimate the magnitude of additive and nonadditive genetic components of resistance and tolerance to spittlebug herbivory, (2) to test for constraints on the evolution of defense by estimating genetic correlations between defense and plant fitness components and (3) to test for constraints on defense strategies by estimating the genetic correlation between resistance and tolerance.

\section{Materials and methods}

\section{Study system}

M. guttatus is an herb native to open, wet habitats throughout much of western North America. It produces many perfect yellow flowers that are typically paired at nodes along erect racemes. Each flower can produce several hundred seeds. M. guttatus is an annual, except in some sites that remain wet year-round, where it can persist throughout the year and spread rhizomatously (Dole, 1992). The mating system of M. guttatus varies from approximately $75 \%$ selfing $(t=0.25)$ to complete outcrossing $(t=1.0)$, with a species mean $t \approx 0.6$ (Ritland and Ritland, 1989; Dudash and Ritland, 1991; Willis, 1993). Inbreeding depression is commonly observed in M. guttatus (Carr and Dudash, 1996; Carr and Eubanks, 2002; Ivey et al. 2004). Voucher specimens of native plants from the population studied herein (M13: Napa County, California, USA, $38^{\circ} 33^{\prime} \mathrm{N}, 122^{\circ} 22^{\prime} \mathrm{W}$ ) are housed in the Illinois Natural History Survey herbarium.

The meadow spittlebug, P. spumarius, is a widespread generalist herbivore that feeds on xylem (Weaver and King, 1954). Spittlebugs were the most abundant herbivore species in our coastal California study sites. Infested plants typically hosted 1-2 spittlebug nymphs, and fewer than half of plants hosted spittlebugs (CT Ivey and DE Carr, personal observation). A single spittlebug nymph can exact significant costs to the survival and reproductive success of $M$. guttatus in the field (Ivey et al., 2004). Because spittlebug attack is costly to plant fitness and attack rates are variable among plants, the potential for some mechanism of defense to evolve seems likely. We were not aware of specific traits that confer antibiosis resistance or tolerance to spittlebug attack in M. guttatus, instead, we characterized defense phenotype (resistance and tolerance) using plant and insect performance traits (see below). The spittlebugs used in this experiment originated from a population in Marin County, CA, USA $\left(38^{\circ} 07^{\prime} \mathrm{N}, 122^{\circ} 56^{\prime} \mathrm{W}\right)$.

\section{Diallel design}

One plant was grown from each of 15 seed families collected at the native site. These 15 parental plants were hand-pollinated in a complete diallel design, in which each parent served as both male and female in all possible pairwise crosses. Flowers serving as pollen recipients were emasculated in bud to prevent selfpollination. A single anther from flowers serving as pollen donors was pressed into the stigma of pollen recipient to perform hand-pollinations. Each fruit matured resulted in several hundred full-sibling seeds. Approximately 50 seeds from each cross were sown into separate 71-mm pots, from which 12 emerging seedlings were randomly chosen to include in the diallel experiment, resulting in a total of 2700 plants. Seedlings were transplanted at the cotyledon stage into individual 71-mm pots. Two plants from each cross were randomly assigned to positions within each of six greenhouse benches (blocks), and one plant from each pair was randomly assigned to receive a second instar spittlebug nymph. Spittlebugs were applied to plants during the rosette stage, which is the developmental stage of M. guttatus during early spring when spittlebug colonization typically occurs. Spittlebugs generally began to feed on plants within minutes of application, and they were allowed to feed until they emerged as adults. Adult spittlebugs were collected at emergence, dried at $50{ }^{\circ} \mathrm{C}$ for 2 days, and weighed on a Cahn C-31 microbalance to the nearest $0.001 \mathrm{mg}$. We recorded the number of days after transplanting when each plant opened its first flower (days to first flower, DFF). After all spittlebugs had been collected, we counted the number of flowers produced by each plant. In addition, all above-ground plant biomass was dried to constant weight at $50{ }^{\circ} \mathrm{C}$ and weighed to the nearest $0.01 \mathrm{~g}$.

\section{Analyses}

To correct for heteroskedasticity, plant and insect biomass data were natural log-transformed and count data (DFF, number of flowers and number of days to spittlebug maturation) were squareroot transformed before the analyses. The effect of spittlebugs on plant biomass and flower number was evaluated using mixedmodel analysis of variance in which dam and block were random effects and herbivory treatment was the fixed effect.

Plant performance (number of flowers and plant biomass) was used to estimate plant tolerance $(T)$ to herbivory as $T=D-U$, where $D=$ fitness of the damaged plant and $U=$ fitness of the undamaged plant (Strauss and Agrawal, 1999). Negative values thus suggest a fitness cost to herbivory, whereas positive values indicate overcompensation. $T$ was calculated for each full-sib pair from each cross within a block, and transformed fitness values were used in calculations to account for differences among families in absolute fitness values. This estimate of tolerance is thus comparable to the proportional fitness of damaged plants (for example, Strauss and Agrawal, 1999). DFF was not used in tolerance calculations because this trait was not significantly altered by spittlebug herbivory (see below).

To estimate plant resistance to spittlebug herbivory, we measured insect performance (number of days to emergence and adult biomass), which we interpreted as reflecting antibiosis components of plant resistance (Painter, 1958). Plants on which insects took longer to mature or on which insects were smaller at maturity were considered to be more resistant.

For each of the six plant traits (three traits measured in each of two herbivory environments), the two insect performance traits, as well as the two tolerance measures, we estimated the magnitude of variation attributable to five genetic sources. This was accomplished by partitioning phenotypic variation into its causal genetic 
components following the 'bio' model of Cockerham and Weir (1977). The five sources of genetic variation include additive effects of genes $\left(V_{\mathrm{A}}\right)$, dominance $\left(V_{\mathrm{D}}\right)$, nonnuclear maternal effects $\left(V_{\mathrm{M}}\right)$, non-nuclear paternal effects $\left(V_{\mathrm{P}}\right)$ and nuclear $\times$ extranuclear interactions between parents $\left(V_{\mathrm{I}}\right)$. The remaining variation observed was attributed to random environmental effects $\left(V_{\mathrm{E}}\right)$. We calculated narrow-sense heritability as $h^{2}=V_{\mathrm{A}} / V_{\mathrm{T}}$, where $V_{\mathrm{T}}$ is total phenotypic variance. We partitioned phenotypic variance into the genetic components, using a computer program written by RG Shaw and FH Shaw (Quercus: see http://www.cbs.umn.edu/eeb/events / quercus.shtml) that uses restricted maximum-likelihood generalized linear models. To test whether the variance components contributed significantly to the observed phenotypic variation, we constrained each component estimate in turn to 0 , reran the model and compared the resulting likelihood values using a log-likelihood ratio test. Two times the difference between the log-likelihood for the constrained model and that for the full model was compared with a $\chi^{2}$ distribution with $\mathrm{df}=1$ (Shaw, 1987).

We constructed bivariate models in Quercus to estimate genetic covariances, from which we inferred genetic costs and tradeoffs associated with herbivory defense. For all bivariate models, we constrained to 0 any variance components estimated as 0 in the univariate models. The significance of covariance estimates was evaluated using log-likelihood ratio tests. To examine the cost of tolerance, we calculated the genetic correlation between estimates of tolerance $(T)$ and the corresponding performance trait on control plants. To evaluate a cost of

Table 1 Mean, coefficient of variation and minimum-maximum values of plant performance of Mimulus guttatus plants (grown with (Spittlebug) or without (Control) Philaenus spumarius nymphs) and performance of spittlebugs feeding on $M$. guttatus grown in a full diallel breeding design

\begin{tabular}{llccc}
\hline Organism & \multicolumn{1}{c}{ Trait } & Mean & CV & Min-max \\
\hline \multirow{2}{*}{ Plants (control) } & Days to first flower & 42.6 & 5.6 & $39-64$ \\
& Flower number & 23.6 & 47.4 & $0-104$ \\
& Biomass (g) & 0.31 & 60.8 & $0.01-1.46$ \\
Plants (spittlebug) & Days to first flower & 42.6 & 5.8 & $32-64$ \\
& Flower number & 21.4 & 46.7 & $0-102$ \\
& Biomass (g) & 0.25 & 62.7 & $0.01-1.15$ \\
Spittlebugs & Days to maturity & 19.7 & 12.5 & $12-33$ \\
& Biomass at maturity (mg) & 2.34 & 25.7 & $0.97-4.77$ \\
& & & &
\end{tabular}

resistance, we calculated genetic correlations between insect performance traits and the performance of control plants. For models testing the cost of resistance, we constrained the environmental covariance to 0 , because the measurements involved different plants. We tested for tradeoffs between tolerance and resistance by calculating the genetic correlation between the two estimates of $T$ and the two insect performance traits.

We constructed two additional bivariate models to examine genetic correlations in plant performance between the control and spittlebug environments. A genetic correlation $=1$ in these models would mean that the performance of plant genotypes is ranked equally regardless of spittlebug attack, which suggests no genetic variation in tolerance to herbivory. A genetic correlation $<1$ indicates a genotype $\times$ environment interaction and can be interpreted as evidence for genetic variation in tolerance to herbivory. Environmental covariances for these models were constrained to 0 , because the traits were measured on different plants (Lynch and Walsh, 1998).

\section{Results}

Spittlebugs reduced flower number by $9 \%$ (Table 1; $F_{1,2443}=30.0, P<0.0001$ ), plant biomass by $17 \%$ (Table 1 ; $\left.\mathrm{F}_{1,2444}=82.7, P<0.0001\right)$, but had no significant effect on the number of days to first flower (Table $1 ; F_{1,2441}=0.02$, $P=0.9$ ). Coefficients of variation were highest for flower number and plant biomass, and were not strongly affected by herbivory treatment (Table 1). Insect performance traits and flowering time had lower variability.

We found substantial narrow-sense heritability $\left(h^{2}\right)$ for the number of days to first flower, of a similar magnitude for both control plants and those infested with spittlebugs (Table 2). Estimates of $h^{2}$ for flower number and plant biomass, on the other hand, were not significantly different from zero, regardless of herbivory environment. Non-nuclear maternal effects, on the other hand, contributed significantly to variation in flower number and plant biomass for plants grown in either spittlebug environments. Non-nuclear paternal effects were the largest source of nonenvironmental variation in flower number among control plants, although this was not a significant source of variation in plants attacked by spittlebugs. Interactions between nuclear and cytoplasmic genes were a small, but significant proportion of phenotypic variation in nearly all plant performance traits.

Table 2 Genetic variance components of life history traits in Mimulus guttatus

\begin{tabular}{|c|c|c|c|c|c|c|}
\hline \multirow[t]{2}{*}{ Genetic source } & \multicolumn{2}{|c|}{ Days to first flower } & \multicolumn{2}{|c|}{ Flower number } & \multicolumn{2}{|c|}{ Plant biomass } \\
\hline & Control & Spittlebug & Control & Spittlebug & Control & Spittlebug \\
\hline Additive & $59.1^{* * * *}$ & $56.7^{* * * *}$ & 0.0 & 5.4 & 12.1 & 11.9 \\
\hline Dominance & 0.0 & 7.3 & 0.0 & 0.0 & 0.0 & 0.0 \\
\hline Maternal & 2.8 & 0.0 & $5.9^{* *}$ & $5.7^{* *}$ & $10.8^{* *}$ & $9.6^{* * *}$ \\
\hline Paternal & 1.2 & 0.0 & $7.4^{* *}$ & 2.3 & 5.3 & 1.9 \\
\hline Interaction & $4.4^{* *}$ & $5.2^{* *}$ & $4.7^{* *}$ & 2.4 & $4.4^{* *}$ & $5.3^{* *}$ \\
\hline Total phenotypic variance & 0.0030 & 0.0032 & 1.1962 & 1.0619 & 0.3519 & 0.4153 \\
\hline
\end{tabular}

Percentage of phenotypic variance attributed to sources of genetic variation for traits of Mimulus guttatus grown without (control) or with (spittlebug) a single Philaenus spumarius nymph herbivore in a full diallel breeding design. These traits do not reflect plant defense directly, but were used to evaluate defense phenotype and its constraints (see text). The additive component represents narrow-sense heritability $\left(h^{2}\right) \times 100$.

${ }^{* *} P<0.01,{ }^{* * *} P<0.001,{ }^{* * *} P<0.0001$ in a log-likelihood ratio test against 0 . 
Table 3 Genetic variance components of herbivory defense in Mimulus guttatus

\begin{tabular}{lcccc}
\hline Genetic source & Days to insect maturity & Adult insect biomass & Tolerance (plant biomass) & Tolerance (flower number) \\
\hline Additive & 1.7 & $11.7^{* *}$ & 0.7 & 0.6 \\
Dominance & 1.4 & 0 & 0 & 0 \\
Maternal & 1.2 & 2.0 & 0 & 0 \\
Paternal & 0 & 0 & 0.1 & $1.3^{*}$ \\
Interaction & 0 & 0 & 0.5197 & 0.5 \\
Total phenotypic variance & 0.0734 & 0.0644 & 1.6428 \\
\hline
\end{tabular}

Percentage of phenotypic variance attributed to sources of genetic variation for the performance of Philaenus spumarius insects feeding on Mimulus guttatus (antibiosis resistance) grown in a diallel design, and for plant tolerance (herbivore-control performance measurements) to insect damage with respect to plant biomass and flower number. Note that the additive component represents narrow-sense heritability $\left(h^{2}\right) \times 100$.

${ }^{*} P<0.05,{ }^{* *} P<0.01$ in a log-likelihood ratio test against 0 .
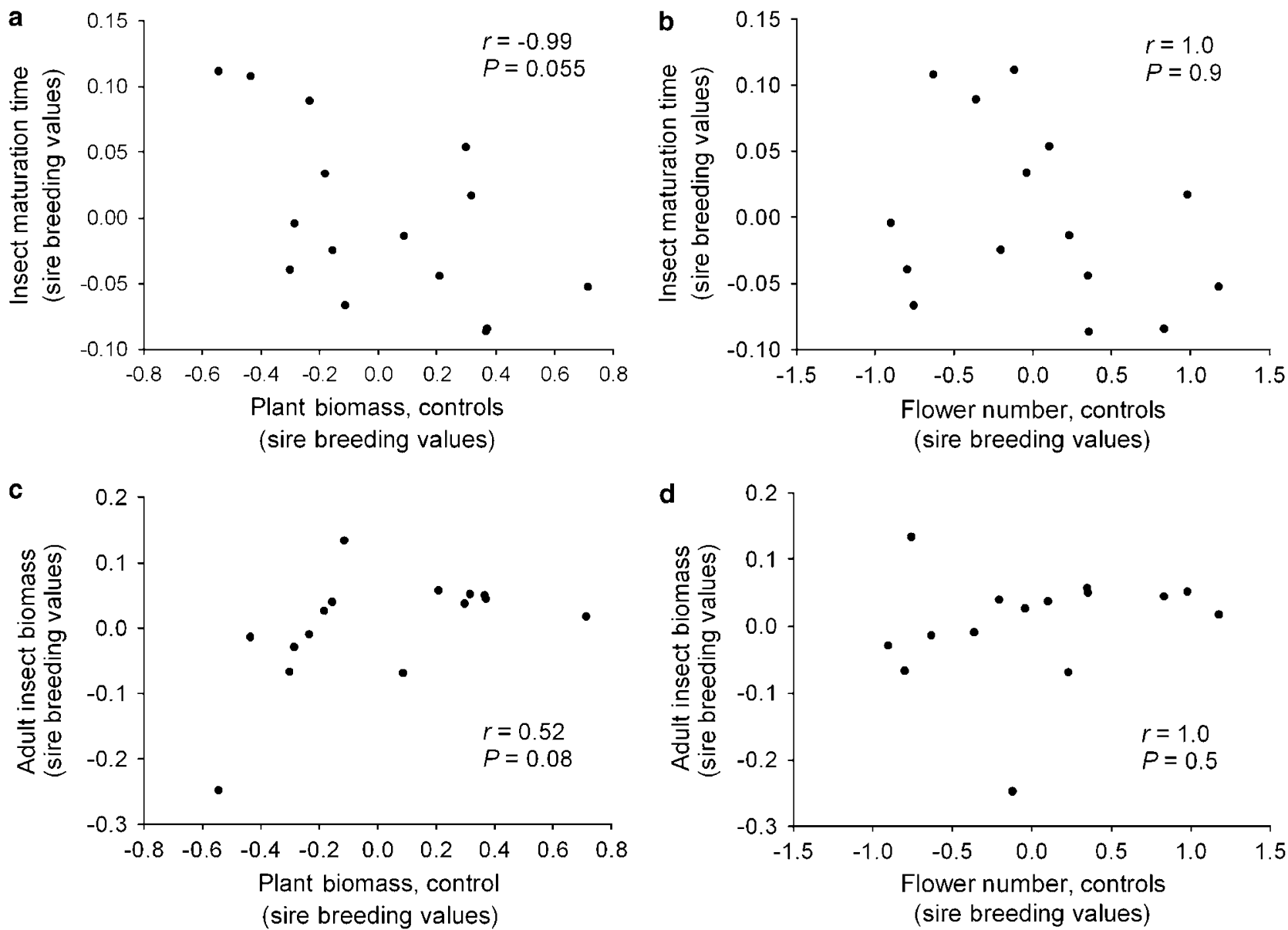

Figure 1 Scatterplots between insect performance, in terms of insect maturation time (a and $\mathbf{b}$ ) and adult insect biomass (c and $\mathbf{d})$ and plant fitness components, in terms of biomass (a and $\mathbf{c}$ ) and flower number (b and $\mathbf{d}$ ) from a diallel breeding design of Mimulus guttatus grown in a greenhouse with and without spittlebug (Philaenus spumarius) nymphs. Insect performance reflects plant antibiosis resistance to herbivory, thus a significant negative ( $(\mathbf{a}$ and $\mathbf{b}$ ) or positive ( $\mathbf{c}$ and $\mathbf{d}$ ) genetic correlation would suggest that the evolution of resistance would be constrained. For illustration, sire breeding values are shown; these were calculated as two times the difference between the sire's mean progeny performance minus that of the overall population (Falconer, 1981). Note that estimates of sire breeding values include some nonadditive sources of genetic variation that were controlled during restricted maximum-likelihood estimation of additive genetic correlations (inset).

No significant proportion of the variation that we observed in emergence time among spittlebugs could be attributed to the genetic sources we partitioned (Table 3). In contrast, we found evidence for significant $h^{2}$ for adult spittlebug biomass, which suggests significant heritability for resistance to herbivory in $M$. guttatus.

Phenotypic variation in tolerance ( $T$ : measured as damaged-undamaged plant performance) was relatively high, ranging from -3.47 to 3.78 for plant biomass and almost twice that, from -6.07 to 4.69 , for flower number. Nonetheless, we found no significant additive genetic variation among plants in $T$, in either measure of plant performance. Non-nuclear paternal effects, however, provided a small, but significant contribution to variation in tolerance based on flower number (Table 3).

Low additive genetic variation in plant performance traits may have limited our power to detect significant additive genetic correlations between traits. Nonetheless, 
some informative trends emerged. We found marginally significant additive genetic correlations between plant performance (biomass) and insect performance (maturation time and biomass at maturity) (Figure 1). The smallest plant genotypes, for example, were those on which spittlebugs took longest to mature (Figure 1a) and those same genotypes produced the smallest spittlebugs (Figure 1c). This may indicate that plant genotypes with greater antibiosis resistance to spittlebugs suffer a fitness cost in terms of biomass.

We found no evidence for a cost of tolerance to herbivory, as measured by the additive genetic correlation between the performance of control plants and tolerance (Figure 2). Maximum-likelihood estimates of additive genetic correlations between tolerance and plant biomass or flower number were both nonsignificant and positive, whereas if tolerance were costly they would be expected to be significantly negative. Genetic correla-
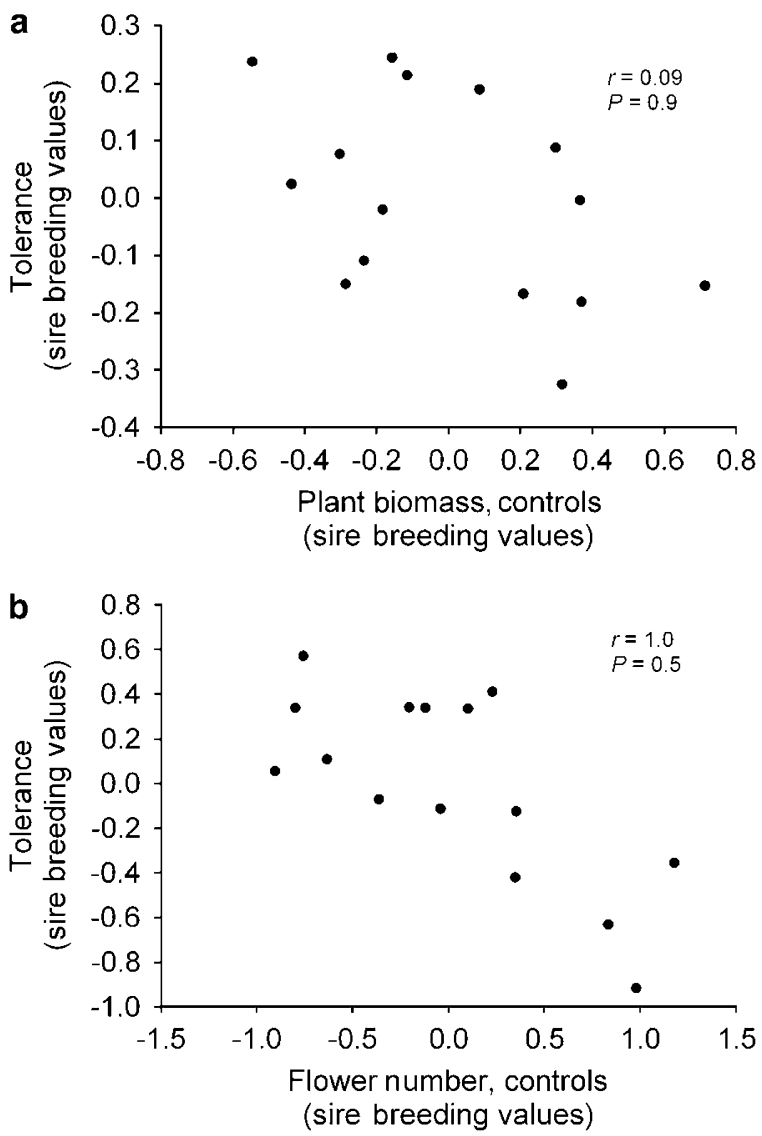

Figure 2 Scatterplots between tolerance to herbivory (calculated as the difference in plant performance between infested and control plants) and performance of control plants with respect to plant biomass (a) and flower number (b). A significant negative genetic correlation would suggest that the evolution of tolerance is constrained by allocation costs. Mimulus guttatus plants were bred in a diallel design, and grown in a greenhouse with and without spittlebug (Philaenus spumarius) nymphs. For illustration, sire breeding values are shown; these were calculated as two times the difference between the sire's mean progeny performance minus that of the overall population (Falconer, 1981). Note that estimates of sire breeding values include some nonadditive sources of genetic variation that were controlled during restricted maximum-likelihood estimation of additive genetic correlations (inset) tions due to nonadditive effects, however, may contribute to constraints on the evolution of tolerance. For example, the correlation between tolerance and plant performance with respect to flower number due to nonnuclear paternal effects was strongly negative $(r=-1.0$, $P=0.003$; Figure $2 b$ ), suggesting that costs of tolerance may be reflected in the characteristics of the pollen donor.

We estimated the additive genetic correlations between plant performance traits across spittlebug environments as a second test for additive genetic variation for tolerance to herbivory. The correlations that we observed were strongly positive and 95\% confidence intervals for the correlations overlapped one in both cases (Figure 3), which reinforces our previous result of low additive genetic variation for $T$ (Table 3 ). A likelihood-ratio test, however, failed to reject the hypothesis that $r=0$, for either correlation (Figure 3), which contradicts the
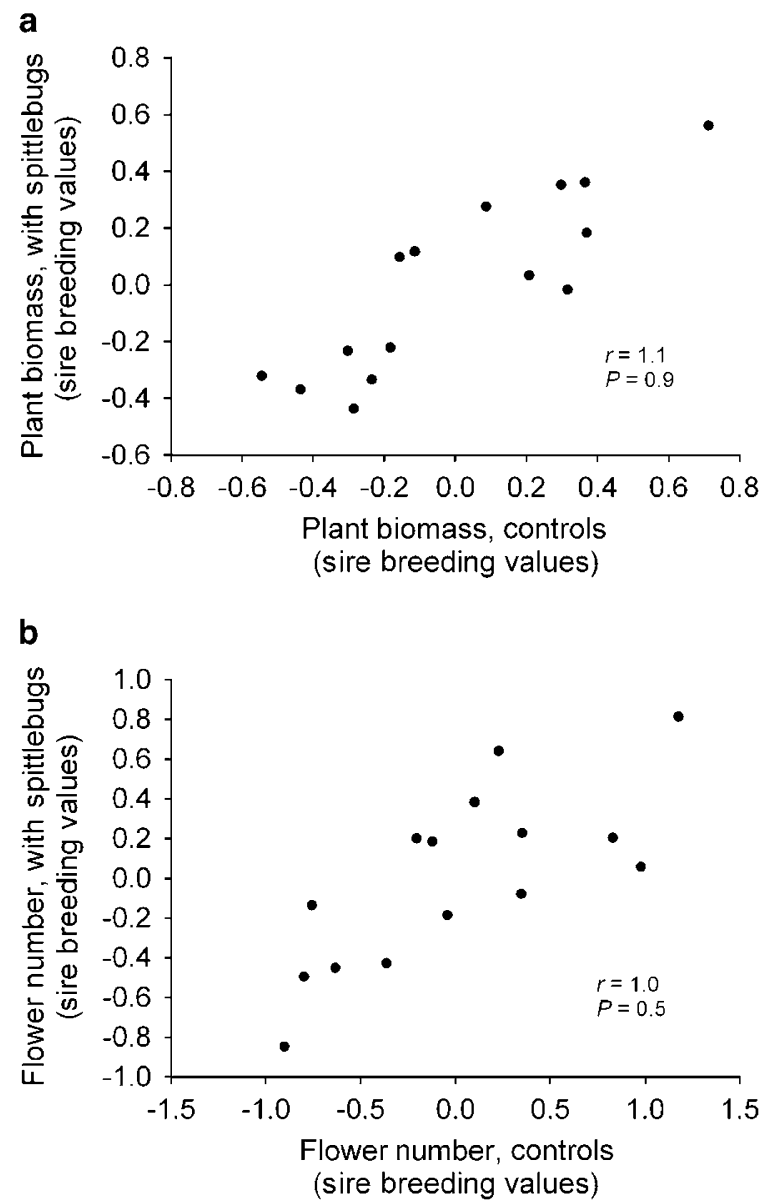

Figure 3 Scatterplots between performance of Mimulus guttatus plants in terms of plant biomass (a) and flower number (b) when grown with or without spittlebug (Philaenus spumarius) nymphs. For illustration, sire breeding values are shown; these were calculated as two times the difference between the sire's mean progeny performance minus that of the overall population (Falconer, 1981) Note that estimates of sire breeding values include some nonadditive sources of genetic variation that were controlled during restricted maximum-likelihood estimation of additive genetic correlations (inset). Approximate standard errors for correlation coefficients are 0.002 and 0.005 for plant biomass and flower number, respectively; confidence intervals $(95 \%)$ for estimates of additive genetic correlation overlapped $r=1.0$ in both cases. See Results for further discussion. 
foregoing result based on confidence intervals. This conflict in test results is likely symptomatic of the low levels of additive genetic variation for the underlying traits, which limits our power to test hypotheses about the strength of genetic correlations between them.

We tested for a tradeoff between defensive strategies to herbivory (tolerance vs resistance) by examining genetic correlations between tolerance and insect performance (Figure 4). Additive genetic correlations between spittlebug maturation time and tolerance were not statistically significant, nor were genetic correlations between adult spittlebug biomass and tolerance.

\section{Discussion}

Although we found variation for defense against spittlebug herbivory in $M$. guttatus, there was little evidence that this variation was genetically based. We found significant heritability for spittlebug biomass, indicating additive genetic variation among plants in resources provided to spittlebugs. Variation in insect performance among plants can reflect variation among plants in resistance to herbivory (Painter, 1958). We also found low, but significant non-nuclear paternal genetic variation for tolerance to herbivory as measured by the difference in flower number between infested and control plants. Nonnuclear paternal effects in plants can stem from a variety of sources, including male gametophytic contributions to endosperm, paternally inherited cytoplasmic genes (for example, cpDNA: Chat et al., 1999), or environmentally induced influences on gene expression or pollen performance (Galloway, 2001); our experiment was not designed to discriminate among these various possibilities. To our knowledge, this is the first study to report nonadditive paternal genetic variation for plant tolerance to herbivory, although substantial reciprocal specific genetic effects for resistance have been reported for some crops (Wilson, 1990; Dhliwayo et al., 2005). With the exception of these two components, however, none of the sources of genetic variation that we examined contributed significantly to the phenotypic variation in defense.

We did not identify traits responsible for herbivore defense in the plants, and instead characterized plant defense indirectly through traits associated with size, fecundity and phenology of plants or insects. Fisher (1930) predicted that life history traits would have little additive genetic variation because of their strong effects on fitness. None of the traits that we measured, except
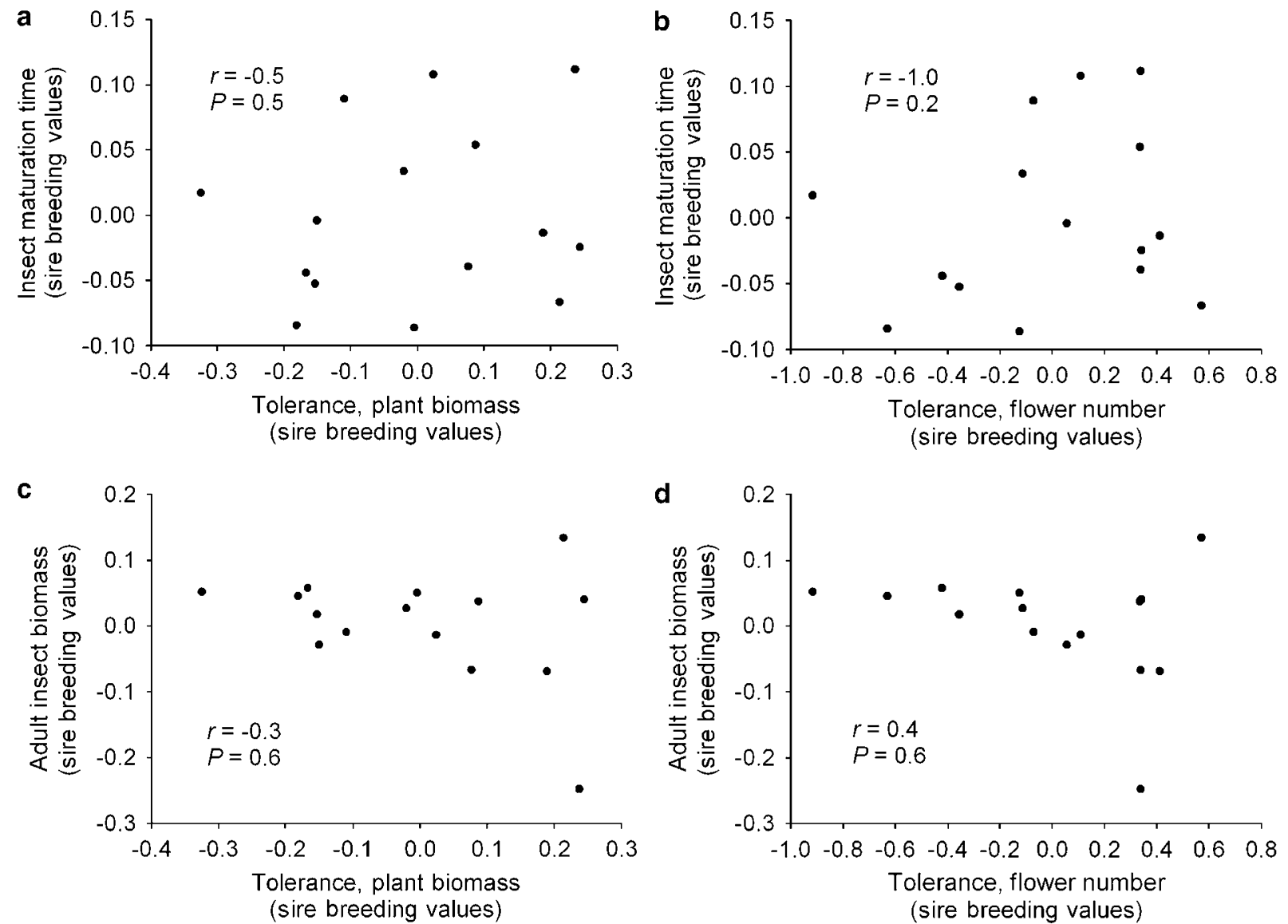

Figure 4 Scatterplots between insect performance, in terms of insect maturation time (a and b) and adult insect biomass (c and d), and tolerance to herbivory (calculated as the difference in plant performance between infested and control plants) with respect to plant biomass (a and $\mathbf{c}$ ) and flower number ( $\mathbf{b}$ and $\mathbf{d}$ ). Insect performance reflects plant antibiosis resistance to herbivory, thus a significant negative (a and b) or positive (c and d) genetic correlation would suggest that a trade-off between the two forms of defense constrains their evolution. Mimulus guttatus plants were bred in a diallel design, and grown in a greenhouse with and without spittlebug (Philaenus spumarius) nymphs. For illustration, sire breeding values are shown; these were calculated as two times the difference between the sire's mean progeny performance minus that of the overall population (Falconer, 1981). Note that estimates of sire breeding values include some nonadditive sources of genetic variation that were controlled during restricted maximum-likelihood estimation of additive genetic correlations (inset). 
flowering time and resistance (measured as insect biomass), had any detectable additive genetic variation, which is consistent with Fisher's (1930) prediction. These results mirror a review of 26 studies estimating $h^{2}$ in plant life history traits in which phenological traits were found to have, on average, higher $h^{2}$ than fecundity or size traits (Mazer and LeBuhn, 1999).

Although heritability was low in most traits measured, the distribution of nonadditive genetic variation in defense traits contrasted with that of the other plant life history traits. Among the defense traits, only one nonadditive component was found to explain a significant proportion of the variation observed (toleranceflower number), whereas genetic variation due to nonnuclear maternal effects, paternal effects and nuclear $\times$ cytoplasmic gene interactions explained a significant amount of phenotypic variation in flowering time, flower number, as well as plant biomass. Depending on the type of effect and its influence on the trait, nonadditive sources of genetic variation such as these can constrain adaptive evolution through generational time lags in response to selection or responses that oppose the direction of selection (Mazer and Gorchov, 1996). Traits under both cytoplasmic and nuclear genetic control, for example, will have a much slower response to selection if there is a negative genetic correlation between the different effects (Roach and Wulff, 1987). An illustrative study of Chamaecrista fasciculata found that interactions between cytoplasmic and nuclear genes influenced seed weight, plant size and fitness in ways that contrasted with nuclear genomic effects and likely limited the plant's adaptive response to local environmental conditions (Galloway and Fenster, 1999). Nonadditive sources of genetic variation may be maintained in traits by fitness effects, by nonreciprocal environment-dependent gene expression, selection among parental lines or other mechanisms (discussed by Mazer and Gorchov, 1996). These nonadditive sources of variation would have been impossible to observe without the diallel breeding design that we used; moreover, with a clonal or nested half-sib design, we would have overestimated the magnitude of heritability in our traits (Lynch and Walsh, 1998). Interestingly, another study involving a different population of $M$. guttatus also reported within-population nonadditive genetic variation for the same traits (Carr et al., 2006), which indicates that complex genetic effects for such traits may be more widespread than previously appreciated. In addition, there was some evidence for environment-dependent expression of nonadditive genetic variation; the presence of spittlebugs reduced the magnitude of nonadditive genetic variation in flower number (Table 2), which suggests that herbivory could dampen the influence of nonadditive genetic variation on natural selection for this trait. In any case, our estimates of genetic variation in tolerance were certainly influenced by the genetic architecture of these underlying traits. Overall, a majority of the phenotypic variation in both tolerance and resistance that we observed, as well as that of the life history traits that we measured, was maintained by environmental sources of variation, which is notable given that the study was conducted in a greenhouse, where environmental variation is expected to be lower than in the field. Environmental conditions can have a strong influence on estimates of genetic variation, and studies conducted in a greenhouse do not always mirror results from a more natural setting.

Although there was phenotypic variation in tolerance, we found no evidence for additive genetic variation in this trait. Genetic variation for herbivory tolerance, however, has been reported for a number of other plant species (Strauss and Agrawal, 1999; Núñez-Farfán et al., 2007), and genetic variation for other traits in M. guttatus is often substantial (Carr and Fenster, 1994; Robertson et al., 1994; Fishman et al., 2002), including other forms of phenotypic plasticity (Murren et al., 2006). Carr et al. (2006), on the other hand, found little evidence for genetic control of tolerance to Cucumber mosaic virus in a different population of $M$. guttatus. The low additive genetic variation for tolerance to spittlebug herbivory in the population we studied would limit its adaptive evolution, even under strong selection. Low levels of additive genetic variation can indicate that selection on a trait has been strong in the past. This would be consistent with Roy and Kirchner's (2000) model of the evolution of tolerance to pathogens; in this model, traits conferring tolerance are expected to sweep to fixation and maintain their advantage through time, because such traits would not affect the prevalence or fitness of the pathogen. This model, however, does not include costs of tolerance, whereas such costs have been reported in other studies of tolerance to herbivory (Stowe et al., 2000). We also found no evidence for dominance variation for tolerance, even though previous experiments, involving this population as well as others, reported considerable variation among families in the response of tolerance to experimental self-fertilization (Carr and Eubanks, 2002; Ivey et al., 2004). The lack of significant dominance variation for tolerance in our diallel cross suggests that these earlier observations were due to mutations unique to family lines (Schultz and Willis, 1995).

We found modest, but significant additive genetic variation among plant genotypes in spittlebug performance (biomass at maturity), which suggests significant heritability for antibiosis resistance. Sire breeding values of spittlebug mass at maturity ranged from 0.78 to $1.14 \mathrm{mg}$, which reflects plant genotypic differences in resources provided to spittlebugs. Phenotypic variation in spittlebug mass at maturity ranged nearly fivefold among individual plants. $M$. guttatus resistance strategies against spittlebugs may also involve antixenosis or traits that influence herbivore preference (for example, Holeski, 2007), although that was not measured in this study.

Plant genotypes that produced smaller spittlebugs (that is, more resistant genotypes) had lower biomass in the absence of spittlebugs, suggesting a fitness cost to resistance. Plant biomass is correlated with total seed mass in natural populations of $M$. guttatus in northern California $(0.68<r<0.80$; Fenster and Ritland, 1994). This genetic correlation may, therefore, constrain the adaptive evolution of resistance to spittlebugs in this population. A positive genetic correlation between control plant size and adult spittlebug size likely reflects the role of plant vigor in spittlebug growth. Vigor has long been recognized to affect plant defense. The plant vigor hypothesis of herbivore resistance (Price, 1991; Hull-Sanders and Eubanks, 2005), for example, predicts herbivore performance to be higher on more vigorous, high-performing plants, which we observed in the positive genetic correlation between size of control plants 
and spittlebug size. We also found a strong negative additive genetic correlation $(r=-0.99, P=0.055)$ between spittlebug development time and size at maturity, which indicated that spittlebugs reared on the smallest (most resistant) plant genotypes were not only smaller but also took longer to mature. We did not find evidence for a fitness benefit of resistance, in that the genetic correlations between spittlebug performance and plant performance in the presence of spittlebugs were not significantly different from zero (data not shown), although such a relationship might be more likely to be observed in field conditions. In contrast to the results for resistance, we found no evidence for a cost of tolerance, based on genetic correlations between tolerance and performance of control plants. Although the genetic correlations between tolerance and plant performance with spittlebugs were positive, as expected if tolerance were adaptive, these were not significantly different from zero. Note, however, that the low additive genetic variation that we observed for tolerance probably limited our power to detect significant genetic correlations. In fact, the only significant genetic variance component that we detected for tolerance was a small paternal effect, and the paternal genetic correlation between tolerance and flower number in control plants was significantly negative, as expected, if tolerance were costly. This suggests that environmental or non-nuclear genetic characteristics of sires that improve tolerance could be constrained by a fitness disadvantage with respect to paternal influences on flower number.

We found no evidence for a tradeoff between tolerance and resistance to spittlebugs in this population. A tradeoff between resistance and tolerance is expected when investment in defense strategies has negative fitness consequences, due to redundancy (van der Meijden et al., 1988). Our results suggest that any fitness costs of tolerance and resistance are not equivalent in $M$. guttatus, which would limit the opportunity for tradeoffs to arise. Similarly, Carr et al. (2006) found no evidence for tradeoffs between tolerance and resistance to Cucumber mosaic virus in M. guttatus. The potential costs that we examined were presumably associated with constraints on resource allocation to other functions, specifically growth or reproduction. Limited evidence for significant genetic correlations between these strategies could indicate that variation in resource acquisition is a more important constraint than variation in allocation to defense (Houle, 1991). Ecological costs, imposed by interactions with other species, may also constrain defense evolution (Strauss et al., 2002). Alternatively, defense against spittlebugs in $M$. guttatus might not be constrained by tradeoffs between tolerance and resistance. A meta-analysis of 31 studies, involving both crops and wild species, found that the genetic correlation between tolerance and resistance did not differ significantly from zero, suggesting that generalized strategies involving both forms of defense may be common (Leimu and Koricheva, 2006). Indeed, Fornoni et al. (2004) showed that a mixed defense strategy, in which resources are allocated to both tolerance and resistance, can be evolutionarily stable where costs of defense vary spatially (see also Núñez-Farfán et al., 2007).

In conclusion, our results suggest that $M$. guttatus has very little available genetic variation for evolving defense against spittlebug herbivory, despite fairly wide pheno- typic variation in resistance and tolerance. Increased tolerance to spittlebug herbivory would not likely evolve in $M$. guttatus due to limited additive genetic variation. Furthermore, increased resistance is unlikely to evolve because of both limited genetic variations and due to high allocation costs for this form of defense. We found no evidence that tolerance and resistance represent mutually exclusive defense strategies, however, suggesting that genetic correlation between tolerance and resistance does not restrict the evolution of a mixed defense strategy (Mauricio, 2000; Núñez-Farfán et al., 2007; Stevens et al., 2007).

\section{Acknowledgements}

We thank S Keller for greenhouse assistance; S Blackwell for field assistance; J Etterson, J Fry, T Juenger and R Shaw for advice on analyses; Wantrup Reserve for logistical support; Point Reyes National Seashore for access to field sites; and two anonymous reviewers for guidance on improving the manuscript. Support was provided by US National Science Foundation Grants DEB-0075225 to DEC and DEB-0074556 to MDE, the Blandy Experimental Farm at the University of Virginia, and the Department of Entomology and Plant Pathology at Auburn University.

\section{References}

Berenbaum MR, Zangerl AR (1992). Genetics of secondary metabolism and herbivore resistance in plants. In: Rosenthal GA, Berenbaum MR (eds). Herbivores: Their Interactions With Secondary Plant Metabolites. Vol. II. Ecological and Evolutionary Processes. Academic Press: San Diego, California, pp 415-438.

Carr DE, Dudash MR (1996). Inbreeding depression in two species of Mimulus (Scrophulariaceae) with contrasting mating systems. Am J Bot 83: 586-593.

Carr DE, Eubanks MD (2002). Inbreeding alters resistance to insect herbivory and host plant quality in Mimulus guttatus (Scrophulariaceae). Evolution 56: 22-30.

Carr DE, Fenster CB (1994). Levels of genetic variation and covariation for Mimulus (Scrophulariaceae) floral traits. Heredity 72: 606-618.

Carr DE, Murphy JF, Eubanks MD (2006). Genetic variation and covariation for resistance and tolerance to Cucumber mosaic virus in Mimulus guttatus (Phrymaceae): a test for costs and restraints. Heredity 96: 29-38.

Chat J, Chalak L, Petit RJ (1999). Strict paternal inheritance of chloroplast DNA and maternal inheritance of mitochondrial DNA in intraspecific crosses of kiwifruit. Theor Appl Genet 99: 314-322.

Cockerham CC, Weir BS (1977). Quadratic analyses of reciprocal crosses. Biometrics 33: 187-203.

Dhliwayo T, Pixley KV, Kazembe V (2005). Combining ability for resistance to maize weevil among 14 Southern African maize inbred lines. Crop Sci 45: 662-667.

Dole JA (1992). Reproductive assurance mechansims in three taxa of the Mimulus guttatus complex (Scrophulariaceae). Am $J$ Bot 79: 650-659.

Dudash MR, Ritland K (1991). Multiple paternity and selffertilization in relation to floral age in Mimulus guttatus (Scrophulariaceae). Am J Bot 78: 1746-1753.

Falconer DS (1981). Introduction to quantitative genetics. 2nd ed Longman Press: London, England, UK.

Fenster CB, Ritland K (1994). Evidence for natural selection on mating system in Mimulus (Schrophulariaceae). Int J Plant Sci 155: 588-596. 
Fineblum WL, Rausher MD (1995). Tradeoff between resistance and tolerance to herbivore damage in a morning glory. Nature 377: 517-520.

Fisher RA (1930). The genetical theory of natural selection. Clarendon Press: Oxford, UK.

Fishman L, Kelly AJ, Willis JH (2002). Minor quantitative trait loci underlie floral traits associated with mating system divergence in Mimulus. Evolution 56: 2138-2155.

Fornoni J, Núñez-Farfán J, Valverde PL, Rausher MD (2004). Evolution of mixed strategies of plant defense allocation against natural enemies. Evolution 58: 1685-1695.

Fornoni J, Valverde PL, Núñez-Farfán J (2003). Quantitative genetics of plant tolerance and resistance against natural enemies of two natural populations of Datura stramonium. Evol Ecol Res 5: 1049-1065.

Galloway LF (2001). Parental environmental effects on life history in the herbaceous plant Campanula americana. Ecology 82: 2781-2789.

Galloway LF, Fenster CB (1999). The effect of nuclear and cytoplasmic genes on fitness and local adaptation in an annual legume, Chamaecrista fasciculata. Evolution 53: $1734-1743$.

Holeski LM (2007). Within and between generation phenotypic plasticity in trichome density of Mimulus guttatus. J Evol Biol 20: 2092-2100.

Houle D (1991). Genetic covariance of fitness correlates: what genetic correlations are made of and why it matters. Evolution 45: 630-648.

Hull-Sanders H, Eubanks MD (2005). Plant defense theory provides insight into interactions involving inbred plants and insect herbivores. Ecology 86: 897-904.

Ivey CT, Carr DE, Eubanks MD (2004). Effects of inbreeding in Mimulus guttatus on tolerance to herbivory in natural environments. Ecology 85: 567-574.

Juenger T, Bergelson J (2000). The evolution of compensation to herbivory in scarlet gilia, Ipomopsis aggregata: herbivoreimposed natural selection and the quantitative genetics of tolerance. Evolution 54: 764-777.

Kennedy GG, Barbour JD (1992). Resistance variation in natural and managed populations. In: Fritz RS, Simms EL (eds). Plant Resistance to Herbivores and Pathogens: Ecology, Evolution, and Genetics. University of Chicago Press: Chicago, IL, USA, pp 13-41.

Leimu R, Koricheva J (2006). A meta-analysis of tradeoffs between plant tolerance and resistance to herbivores: combining the evidence from ecological and agricultural studies. Oikos 112: 1-9.

Lynch M, Walsh B (1998). Genetics and analysis of quantitative traits. Sinauer: Sunderland, MA, USA.

Maddox GD, Root RB (1987). Resistance to 16 diverse species of herbivorous insects within a population of goldenrod, Solidago altissima: genetic variation and heritability. Oecologia 72: 8-14.

Marquis RJ (1990). Genotypic variation in leaf damage in Piper arieianum (Piperaceae) by a multispecies assemblage of herbivores. Evolution 44: 104-120.

Mauricio R (2000). Natural selection and the joint evolution of tolerance and resistance as plant defenses. Evol Ecol 14: 491-507.

Mazer SJ, Gorchov DL (1996). Parental effects on progeny phenotype in plants: distinguishing genetic and environmental causes. Evolution 50: 44-53.
Mazer SJ, LeBuhn G (1999). Genetic variation in life history traits: heritability estimates within and genetic differentiation among populations. In: Vuorisalo TO, Mutikainen PK (eds). Life History Evolution in Plants.. Kluwer Academic Publishers: Dordrecht, The Netherlands, pp 85-171.

Murren CJ, Douglass L, Gibson A, Dudash MR (2006) Individual and combined effects of $\mathrm{Ca} / \mathrm{Mg}$ ratio and water on trait expression in Mimulus guttatus. Ecology 87: 2591-2602.

Núñez-Farfán J, Fornoni J, Valverde PL (2007). The evolution of resistance and tolerance to herbivores. Annu Rev Ecol Evol S 38: 541-566.

Painter RH (1958). Resistance of plants to insects. Annu Rev Entomol 3: 267-290.

Price PW (1991). The plant vigor hypothesis and herbivore attack. Oikos 62: 244-251.

Ritland C, Ritland K (1989). Variation of sex allocation among eight taxa of the Mimulus guttatus species complex (Scrophulariaceae). Am J Bot 76: 1731-1739.

Roach DA, Wulff RD (1987). Maternal effects in plants. Annu Rev Ecol Syst 18: 209-235.

Robertson AW, Diaz A, MacNair MR (1994). The quantitative genetics of floral characters in Mimulus guttatus. Heredity 72: 300-311.

Roy BA, Kirchner JW (2000). Evolutionary dynamics of pathogen resistance and tolerance. Evolution 54: 51-63.

Schultz ST, Willis JH (1995). Individual variation in inbreeding depression: the roles of inbreeding history and mutation. Genetics 141: 1209-1223.

Shaw RG (1987). Maximum-likelihood approaches applied to quantitative genetics of natural populations. Evolution 41: 812-826.

Simms EL, Rausher MD (1987). Costs and benefits of plant resistance to herbivory. Am Nat 130: 570-581.

Soper JF, McIntosh MS, Elden TC (1984). Diallel analysis of potato leafhopper resistance among selected alfalfa clones. Crop Sci 24: 667-670.

Stevens MT, Waller DM, Lindroth RL (2007). Resistance and tolerance in Populus tremuloides: genetic variation, costs, and environmental dependency. Evol Ecol 21: 829-847.

Stowe KA, Marquis RJ, Hochwender CG, Simms EL (2000). The evolutionary ecology of tolerance to consumer damage. Annu Rev Ecol Syst 31: 565-595.

Strauss SY, Agrawal AA (1999). The ecology and evolution of plant tolerance to herbivory. Trends Ecol Evol 14: 179-185.

Strauss SY, Rudgers JA, Lau JA, Irwin RE (2002). Direct and ecological costs of resistance to herbivory. Trends Ecol Evol 17: 278-285.

van der Meijden E, Wijn M, Verkaar HJ (1988). Defense and regrowth: alternative plant strategies in the struggle against herbivores. Oikos 51: 355-363.

Weaver CR, King DR (1954). Meadow spittlebug. Research Bulletin no. 741. Ohio Agricultural Experiment Station: Wooster, Ohio.

Willis JH (1993). Partial self-fertilization and inbreeding depression in two populations of Mimulus guttatus. Heredity 71: 145-154.

Wilson FD (1990). Relative resistance of cotton lines to pink bollworm. Crop Sci 30: 500-504.

Wise MJ (2007). Evolutionary ecology of resistance to herbivory: an investigation of potential genetic constraints in the multiple-herbivore community of Solanum carolinense. New Phytol 175: 773-784. 